\title{
New inequalities for hyperbolic functions and their applications
}

Ling Zhu*

\section{"Correspondence:}

zhuling0571@163.com

Department of Mathematics,

Zhejiang Gongshang University,

Hangzhou, Zhejiang 310018,

P.R. China

\begin{abstract}
In this paper, we obtain some new inequalities in the exponential form for the whole of the triples about the four functions $\{1,(\sinh t) / t$, $\exp (t \operatorname{coth} t-1), \cosh t\}$. Then we generalize some well-known inequalities for the arithmetic, geometric, logarithmic, and identric means to obtain analogous inequalities for their $p$ th powers, where $p>0$. MSC: 26E60; 26D07
\end{abstract}

Keywords: hyperbolic sine; hyperbolic cosine; hyperbolic cotangent; geometric mean; logarithmic mean; identric mean; arithmetic mean; best constants

\section{Introduction}

Let $\sinh t, \cosh t$, and $\operatorname{coth} t$ be the hyperbolic sine, hyperbolic cosine, and hyperbolic cotangent, respectively. It is well known that (see [1-6])

$$
1<\frac{\sinh t}{t}<e^{t \operatorname{coth} t-1}<\cosh t
$$

holds for all $t \neq 0$.

In the recent paper [7], we have established the following Cusa-type inequalities of exponential type for the triple $\{1,(\sinh t) / t, \cosh t\}$ described as follows.

Theorem 1.1 (Cusa-type inequalities [7, Part (i) of Theorem 1.1]) Let $p \geq 4 / 5$, and $t \neq 0$. Then the double inequality

$$
(1-\lambda)+\lambda(\cosh t)^{p}<\left(\frac{\sinh t}{t}\right)^{p}<(1-\eta)+\eta(\cosh t)^{p}
$$

holds if and only if $\eta \geq 1 / 3$ and $\lambda \leq 0$.

On the other hand, the author of this paper [8] obtains the following inequalities of exponential type for the triple $\{1, \exp (t \operatorname{coth} t-1), \cosh t\}$.

Theorem $1.2([8$, Theorem 2]) Let $p>0$, and $t \neq 0$. Then

(1) if $0<p \leq 6 / 5$, the double inequality

$$
\alpha(\cosh t)^{p}+(1-\alpha)<e^{p(t \operatorname{coth} t-1)}<\beta(\cosh t)^{p}+(1-\beta)
$$

holds if and only if $\alpha \leq 2 / 3$ and $\beta \geq(2 / e)^{p}$; 
(2) if $p \geq 2$, the double inequality

$$
\alpha(\cosh t)^{p}+(1-\alpha)<e^{p(t \operatorname{coth} t-1)}<\beta(\cosh t)^{p}+(1-\beta)
$$

holds if and only if $\alpha \leq(2 / e)^{p}$ and $\beta \geq 2 / 3$.

Next, we do the work for each of the triples $\{(\sinh t) / t, \exp (t \operatorname{coth} t-1), \cosh t\}$ and $\{1,(\sinh t) / t, \exp (t \operatorname{coth} t-1)\}$, and obtain the following two new results.

Theorem 1.3 Let $0<p \leq 8 / 5$, and $t \neq 0$. Then

$$
\alpha(\cosh t)^{p}+(1-\alpha)\left(\frac{\sinh t}{t}\right)^{p}<e^{p(t \operatorname{coth} t-1)}<\beta(\cosh t)^{p}+(1-\beta)\left(\frac{\sinh t}{t}\right)^{p}
$$

holds if and only if $\alpha \leq 1 / 2$ and $\beta \geq(2 / e)^{p}$

Theorem 1.4 Let $p \geq 286 / 693$, and $t \neq 0$. Then

$$
\alpha+(1-\alpha) e^{p(t \operatorname{coth} t-1)}<\left(\frac{\sinh t}{t}\right)^{p}<\beta+(1-\beta) e^{p(t \operatorname{coth} t-1)}
$$

holds if and only if $\beta \leq 1 / 2$ and $\alpha \geq 1$.

In this paper, we shall give the elementary proofs of Theorem 1.3 and Theorem 1.4. In the last section, we apply Theorems 1.1-1.4 to obtain some new results for four classical means.

\section{Lemmas}

Lemma $2.1([9-11])$ Let $f, g:[a, b] \rightarrow \mathbb{R}$ be two continuous functions which are differentiable on $(a, b)$. Further, let $g^{\prime} \neq 0$ on $(a, b)$.Iff $/ g^{\prime}$ is increasing (or decreasing) on $(a, b)$, then the functions $\left(f(x)-f\left(b^{-}\right)\right) /\left(g(x)-g\left(b^{-}\right)\right)$and $\left(f(x)-f\left(a^{+}\right)\right) /\left(g(x)-g\left(a^{+}\right)\right)$are also increasing (or decreasing) on $(a, b)$.

Lemma 2.2 Let $t \in(0,+\infty)$. Then the inequality

$$
D(t) \triangleq t \sinh ^{5} t+2 t \sinh ^{3} t+t^{4} \cosh t-\sinh ^{4} t \cosh t-t^{3} \sinh ^{3} t-2 t^{3} \sinh t>0
$$

holds.

Proof Using the power series expansions of the functions $\sinh ^{5} t, \sinh ^{3} t, \cosh t, \sinh ^{4} t \times$ $\cosh t$, and $\sinh t$, we have

$$
\begin{aligned}
D(t)= & \frac{1}{16} t(\sinh 5 t-5 \sinh 3 t+10 \sinh t)+\frac{1}{2} t(\sinh 3 t-3 \sinh t)+t^{4} \cosh t \\
& -\frac{1}{16}(\cosh 5 t-3 \cosh 3 t+2 \cosh t)-\frac{1}{4} t^{3}(\sinh 3 t-3 \sinh t)-2 t^{3} \sinh t \\
= & \frac{1}{16} \sum_{n=0}^{\infty} \frac{5^{2 n+1}-5 \cdot 3^{2 n+1}+10}{(2 n+2) !} t^{2 n+1}+\frac{1}{2} \sum_{n=0}^{\infty} \frac{3^{2 n+1}-3}{(2 n+1) !} t^{2 n+2}+\sum_{n=0}^{\infty} \frac{1}{(2 n) !} t^{2 n+4}
\end{aligned}
$$




$$
\begin{aligned}
& -\frac{1}{16} \sum_{n=0}^{\infty} \frac{5^{2 n}-3 \cdot 3^{2 n}+2}{(2 n) !} t^{2 n}-\frac{1}{4} \sum_{n=0}^{\infty} \frac{3^{2 n+1}-3}{(2 n+1) !} t^{2 n+4}-2 \sum_{n=0}^{\infty} \frac{1}{(2 n+1) !} t^{2 n+4} \\
= & \frac{1}{16} \sum_{n=3}^{\infty} \frac{l_{n}}{(2 n+4) !} t^{2 n+4},
\end{aligned}
$$

where

$$
\begin{aligned}
l_{n}= & (2 n+4)\left(5^{2 n+3}-5 \cdot 3^{2 n+3}+10\right)+8(2 n+4)\left(3^{2 n+3}-3\right) \\
& +16(2 n+4)(2 n+3)(2 n+2)(2 n+1)-\left(5^{2 n+4}-3 \cdot 3^{2 n+4}+2\right) \\
& -4(2 n+4)(2 n+3)(2 n+2)\left(3^{2 n+1}-3\right)-32(2 n+4)(2 n+3)(2 n+2) \\
= & (250 n-125) 25^{n}+\left(279-462 n-432 n^{2}-96 n^{3}\right) 9^{n} \\
& +256 n^{4}+1,120 n^{3}+1,520 n^{2}+532 n-154, \quad n=3,4, \ldots
\end{aligned}
$$

Using a basic differential method, we can easily prove

$$
\begin{aligned}
f(x) \triangleq & (250 x-125) 25^{x}+\left(279-462 x-432 x^{2}-96 x^{3}\right) 9^{x} \\
& +256 x^{4}+1,120 x^{3}+1,520 x^{2}+532 x-154>0
\end{aligned}
$$

on $[3, \infty)$. This leads to $l_{n}>0$ for $n=3,4, \ldots$, and $D(t)>0$. So, the proof of Lemma 2.2 is complete.

\section{Proof of Theorem 1.3}

Let

$$
F(t) \equiv \frac{\left(\frac{t}{\sinh t} e^{t \operatorname{coth} t-1}\right)^{p}-1}{(t \operatorname{coth} t)^{p}-1}=\frac{f_{1}(t)-f_{1}\left(0^{+}\right)}{g_{1}(t)-g_{1}\left(0^{+}\right)},
$$

where $f_{1}(t)=\left(\frac{t}{\sinh t} e^{t \operatorname{coth} t-1}\right)^{p}$ and $g_{1}(t)=(t \operatorname{coth} t)^{p}$. Then

$$
k_{1}(t) \triangleq \frac{f_{1}^{\prime}(t)}{g_{1}^{\prime}(t)}=\frac{e^{p(t \operatorname{coth} t-1)}}{(\cosh t)^{p-1}} \cdot \frac{\sinh ^{2} t-t^{2}}{\sinh t(\sinh t \cosh t-t)} .
$$

We compute

$$
k_{1}^{\prime}(t)=\frac{e^{p(t \operatorname{coth} t-1)}}{(\cosh t)^{p}} \cdot \frac{u_{1}(t)}{(\sinh t)^{3}(\sinh t \cosh t-t)^{2}},
$$

where

$$
\begin{aligned}
u_{1}(t)= & 2 t^{2} \sinh ^{4} t \cosh t+\sinh ^{4} t \cosh t-4 t \sinh ^{5} t \\
& -3 t \sinh ^{3} t+3 t^{2} \sinh ^{2} t \cosh t-t^{3} \sinh t \\
& -p\left(t \sinh ^{5} t+2 t \sinh ^{3} t+t^{4} \cosh t-\sinh ^{4} t \cosh t-t^{3} \sinh ^{3} t-2 t^{3} \sinh t\right) \\
= & 2 t^{2} \sinh ^{4} t \cosh t+\sinh ^{4} t \cosh t-4 t \sinh ^{5} t \\
& -3 t \sinh ^{3} t+3 t^{2} \sinh ^{2} t \cosh t-t^{3} \sinh t-p D(t) .
\end{aligned}
$$


If $0<p \leq 8 / 5$, by Lemma 2.2 we have

$$
\begin{aligned}
5 u_{1}(t) \geq & 10 t^{2} \sinh ^{4} t \cosh t+13 \sinh ^{4} t \cosh t-28 t \sinh ^{5} t \\
& -46 t \sinh ^{3} t+30 t^{2} \sinh ^{2} t \cosh t+6 t^{3} \sinh t-8 t^{4} \cosh t+8 t^{3} \sinh ^{3} t \\
= & \sum_{n=3}^{\infty} \frac{h_{n}}{16(2 n+4) !} t^{2 n+4}
\end{aligned}
$$

where

$$
\begin{aligned}
h_{n}= & 10(2 n+4)(2 n+3)\left(5^{2 n+2}-3 \cdot 3^{2 n+2}+2\right)+13\left(5^{2 n+4}-3 \cdot 3^{2 n+4}+2\right) \\
& -28(2 n+4)\left(5^{2 n+3}-5 \cdot 3^{2 n+3}+10\right)-184(2 n+4)\left(3^{2 n+3}-3\right) \\
& +120(2 n+4)(2 n+3)\left(3^{2 n+2}-1\right)+96(2 n+4)(2 n+3)(2 n+2)(2 n+1) 2 n \\
& -128(2 n+4)(2 n+3)(2 n+2)(2 n+1)+96(2 n+4)(2 n+3)(2 n+2)\left(3^{2 n}-1\right) \\
= & \left(1,000 n^{2}-3,500 n-2,875\right) 25^{n}+\left(768 n^{3}+6,696 n^{2}+13,956 n+4,113\right) 9^{n} \\
& +(2 n+4)(2 n+3)(2 n+2)(2 n+1)(192 n-128) \\
& -96(2 n+4)(2 n+3)(2 n+2)-100(2 n+4)(2 n+3)+272(2 n+4)+26 \\
> & 0
\end{aligned}
$$

for $n=3,4, \ldots$.

We have $u_{1}(t)>0$ for $0<p \leq 8 / 5$. So, $k_{1}^{\prime}(t)>0$ for $t>0$, and $f_{1}^{\prime}(t) / g_{1}^{\prime}(t)=k_{1}(t)$ is increasing on $(0,+\infty)$. Hence, $F(t)$ is increasing on $(0,+\infty)$ by Lemma 2.1. At the same time, $\lim _{t \rightarrow 0^{+}} F(t)=1 / 2$ and $\lim _{t \rightarrow+\infty} F(t)=(2 / e)^{p}$. So, the proof of Theorem 1.3 is complete.

\section{Proof of Theorem 1.4}

Let

$$
S(t) \equiv \frac{\left(\frac{\sinh t}{t} e^{1-t \operatorname{coth} t}\right)^{p}-1}{e^{p(1-t \operatorname{coth} t)}-1}=\frac{f_{2}(t)-f_{2}\left(0^{+}\right)}{g_{2}(t)-g_{2}\left(0^{+}\right)},
$$

where $f_{2}(t)=\left(\frac{\sinh t}{t} e^{1-t \operatorname{coth} t}\right)^{p}$ and $g_{2}(t)=e^{p(1-t \operatorname{coth} t)}$. Then

$$
k_{2}(t) \triangleq \frac{f_{2}^{\prime}(t)}{g_{2}^{\prime}(t)}=\left(\frac{\sinh t}{t}\right)^{p-1} \frac{(\sinh t)^{3}-t^{2} \sinh t}{t^{2}(\sinh t \cosh t-t)},
$$

and

$$
k_{2}^{\prime}(t)=\left(\frac{\sinh t}{t}\right)^{p-2} \frac{u_{2}(t)}{t^{4}(\sinh t \cosh t-t)^{2}},
$$

where

$$
\begin{aligned}
& u_{2}(t) \\
& \quad=\left[t \sinh ^{6} t+2 t \sinh ^{4} t-\sinh ^{5} t \cosh t-t^{3} \sinh ^{4} t-2 t^{3} \sinh ^{2} t+\frac{t^{4}}{2} \sinh 2 t\right](p-1)
\end{aligned}
$$


$+\left(t \sinh ^{6} t+5 t \sinh ^{4} t+t^{3} \sinh ^{4} t-t^{3} \sinh ^{2} t-3 t^{2} \sinh ^{3} t \cosh t+t^{4} \sinh t \cosh t\right.$

$\left.-2 \sinh ^{5} t \cosh t\right)$

$$
=\sum_{n=3}^{\infty}\left[c_{n}(p-1)+d_{n}\right] t^{2 n+5}=\sum_{n=3}^{\infty} c_{n}\left[p-\left(1-\frac{d_{n}}{c_{n}}\right)\right] t^{2 n+5}=\sum_{n=3}^{\infty} c_{n}\left[p-e_{n}\right] t^{2 n+5},
$$

where $e_{n}=1-\left(d_{n} / c_{n}\right)$ and

$$
\begin{aligned}
c_{n}= & \frac{1}{2^{5}} \frac{6^{2 n+4}-6 \cdot 4^{2 n+4}+15 \cdot 2^{2 n+4}}{(2 n+4) !}+\frac{1}{2^{2}} \frac{4^{2 n+4}-4 \cdot 2^{2 n+4}}{(2 n+4) !}-\frac{1}{2^{3}} \frac{4^{2 n+2}-4 \cdot 2^{2 n+2}}{(2 n+2) !} \\
& -\frac{1}{2^{5}} \frac{6^{2 n+5}-4 \cdot 4^{2 n+5}+5 \cdot 2^{2 n+5}}{(2 n+5) !}-\frac{2^{2 n+2}}{(2 n+2) !}+\frac{1}{2} \frac{2^{2 n+1}}{(2 n+1) !}>0, \quad n=3,4, \ldots, \\
d_{n}= & \frac{1}{2^{5}} \frac{6^{2 n+4}-6 \cdot 4^{2 n+4}+15 \cdot 2^{2 n+4}}{(2 n+4) !}+\frac{5}{2^{3}} \frac{4^{2 n+4}-4 \cdot 2^{2 n+4}}{(2 n+4) !}+\frac{1}{2^{3}} \frac{4^{2 n+2}-4 \cdot 2^{2 n+2}}{(2 n+2) !} \\
& -\frac{1}{2^{4}} \frac{6^{2 n+5}-4 \cdot 4^{2 n+5}+5 \cdot 2^{2 n+5}}{(2 n+5) !}-\frac{3}{2^{3}} \frac{4^{2 n+3}-2 \cdot 2^{2 n+3}}{(2 n+3) !}+\frac{1}{2} \frac{2^{2 n+1}}{(2 n+1) !} \\
& -\frac{1}{2} \frac{2^{2 n+2}}{(2 n+2) !}, \quad n=3,4, \ldots
\end{aligned}
$$

Let

$$
\begin{aligned}
j(n)= & -12(2 n+5)\left(4^{2 n+4}-4 \cdot 2^{2 n+4}\right)+\left(6^{2 n+5}-4 \cdot 4^{2 n+5}+5 \cdot 2^{2 n+5}\right) \\
& -8(2 n+5)(2 n+4)(2 n+3)\left(4^{2 n+2}-4 \cdot 2^{2 n+2}\right) \\
& -16(2 n+5)(2 n+4)(2 n+3) 2^{2 n+2}+12(2 n+5)(2 n+4)\left(4^{2 n+3}-2 \cdot 2^{2 n+3}\right) \\
= & 7,776 \cdot 36^{n}+[768(2 n+5)(2 n+4)-128(2 n+5)(2 n+4)(2 n+3) \\
& -3,072(2 n+5)-4,096] 16^{n} \\
& +[64(2 n+5)(2 n+4)(2 n+3)-192(2 n+5)(2 n+4)+768(2 n+5)+160] 4^{n}, \\
i(n)= & (2 n+5)\left(6^{2 n+4}-6 \cdot 4^{2 n+4}+15 \cdot 2^{2 n+4}\right)+8(2 n+5)\left(4^{2 n+4}-4 \cdot 2^{2 n+4}\right) \\
& -\left(6^{2 n+5}+4 \cdot 4^{2 n+5}-5 \cdot 2^{2 n+5}\right)-16(2 n+5)(2 n+4)(2 n+3)\left(4^{2 n+1}-2^{2 n+2}\right) \\
& -32(2 n+5)(2 n+4)(2 n+3) 2^{2 n+2}+32(2 n+5)(2 n+4)(2 n+3)(2 n+2) 2^{2 n} \\
= & (2,592 n-1,296) \cdot 36^{n}+[512(2 n+5)+4,096-64(2 n+5)(2 n+4)(2 n+3)] 16^{n} \\
& +[32(2 n+5)(2 n+4)(2 n+3)(2 n+2)-64(2 n+5)(2 n+4)(2 n+3) \\
& -272(2 n+5)-160] 4^{n} .
\end{aligned}
$$

Then

$$
e_{n}=1-\frac{d_{n}}{c_{n}}=\frac{j(n)}{i(n)} .
$$

Let $\Delta(n)=286 i(n)-693 j(n)$. Then

$$
\begin{aligned}
\Delta(n)= & (741,313 n-5,759,424) 36^{n}+16^{n}[2,275,328(2 n+5)+4,009,984 \\
& +70,400(2 n+5)(2 n+4)(2 n+3)-532,224(2 n+5)(2 n+4)]
\end{aligned}
$$




$$
\begin{aligned}
& +4^{n}[9,152(2 n+5)(2 n+4)(2 n+3)(2 n+2)-62,656(2 n+5)(2 n+4)(2 n+3) \\
& +133,056(2 n+5)(2 n+4)-610,016(2 n+5)-156,640]
\end{aligned}
$$

First, we check that $\Delta(n)>0$ for $n=3,4,5,6,7$; second, we can easily obtain that $\Delta(n)>0$ for $n \geq 8$. So, we have that $\Delta(n)>0$ for $n=3,4, \ldots$.

So, we have $u_{2}(t)>0$ for $p \geq 286 / 693$. So, $k_{2}^{\prime}(t)>0$ for $t>0$, and $f_{2}^{\prime}(t) / g_{2}^{\prime}(t)=k_{2}(t)$ is increasing on $(0,+\infty)$. Hence, $S(t)$ is increasing on $(0,+\infty)$ by Lemma 2.1 when $p \geq 286 / 693$. At the same time, $\lim _{t \rightarrow 0^{+}} S(t)=1 / 2$ and $\lim _{t \rightarrow+\infty} S(t)=1$. So, the proof of Theorem 1.4 is complete.

\section{Applications of theorems}

In this section, we assume that $x$ and $y$ are two different positive numbers. Let $A(x, y)$, $G(x, y), L(x, y)$, and $I(x, y)$ be the arithmetic, geometric, logarithmic, and identric means, respectively. Without loss of generality, we set $0<x<y$. By the transformation $t=$ $(\log (y / x)) / 2$, we can compute and obtain

$$
\begin{aligned}
& \frac{L(x, y)}{G(x, y)}=\frac{\sinh t}{t}, \\
& \frac{I(x, y)}{G(x, y)}=e^{t \operatorname{coth} t-1}, \\
& \frac{A(x, y)}{G(x, y)}=\cosh t,
\end{aligned}
$$

where $t>0$.

Now, the four results in Section 1 are equivalent to the following ones for four classical means.

Theorem 5.1 Let $p \geq 4 / 5$, and $x$ and $y$ be positive real numbers with $x \neq y$. Then

$$
\alpha A^{p}(x, y)+(1-\alpha) G^{p}(x, y)<L^{p}(x, y)<\beta A^{p}(x, y)+(1-\beta) G^{p}(x, y)
$$

holds if and only if $\alpha \leq 0$ and $\beta \geq 1 / 3$.

Theorem 5.1 can deduce the following one, which is from Zhu [8].

Corollary $5.2([8$, Theorem 1$])$ Let $p \geq 1$, and $x$ and $y$ be positive real numbers with $x \neq y$. Then

$$
\alpha A^{p}(x, y)+(1-\alpha) G^{p}(x, y)<L^{p}(x, y)<\beta A^{p}(x, y)+(1-\beta) G^{p}(x, y)
$$

holds if and only if $\alpha \leq 0$ and $\beta \geq 1 / 3$.

When letting $p=1$ in Theorem 5.1, one can obtain the result (see [12-14], [15, Theorem 1]).

Corollary 5.3 Let $x$ and $y$ be positive real numbers with $x \neq y$. Then

$$
\alpha A(x, y)+(1-\alpha) G(x, y)<L(x, y)<\beta A(x, y)+(1-\beta) G(x, y)
$$

holds if and only if $\alpha \leq 0$ and $\beta \geq 1 / 3$. 
When letting $\beta=1 / 3$ in the right-hand inequality of (5.3), one can obtain the well-known inequality by Carlson [16]

$$
L(x, y)<\frac{1}{3} A(x, y)+\frac{2}{3} G(x, y) .
$$

Theorem 5.4 Let $p>0$. Then

(1) if $0<p \leq 6 / 5$, the double inequality

$$
\alpha A^{p}(x, y)+(1-\alpha) G^{p}(x, y)<I^{p}(x, y)<\beta A^{p}(x, y)+(1-\beta) G^{p}(x, y)
$$

holds if and only if $\alpha \leq 2 / 3$ and $\beta \geq(2 / e)^{p}$;

(2) if $p \geq 2$, the double inequality

$$
\alpha A^{p}(x, y)+(1-\alpha) G^{p}(x, y)<I^{p}(x, y)<\beta A^{p}(x, y)+(1-\beta) G^{p}(x, y)
$$

holds if and only if $\alpha \leq(2 / e)^{p}$ and $\beta \geq 2 / 3$.

The part (2) of Theorem 5.4 is a result of Trif [17].

When letting $p=2$ and $\beta=2 / 3$ in the right-hand inequality of (5.6), one can obtain the following result, which is from Sándor and Trif [18].

$$
I^{2}(x, y)<\frac{2}{3} A^{2}(x, y)+\frac{1}{3} G^{2}(x, y) .
$$

When letting $p=1$ in the double inequality (5.5), one can obtain the following result (see [12], [15, Theorem 2]).

Corollary 5.5 Let $x$ and $y$ be positive real numbers with $x \neq y$. Then

$$
\alpha A(x, y)+(1-\alpha) G(x, y)<I(x, y)<\beta A(x, y)+(1-\beta) G(x, y)
$$

holds if and only if $\alpha \leq 2 / 3$ and $\beta \geq 2 / e$.

When letting $\alpha=2 / 3$ in the left-hand inequality in (5.8), one can obtain the following result, which is from Sándor [19].

$$
\frac{2}{3} A(x, y)+\frac{1}{3} G(x, y)<I(x, y) .
$$

Theorem 5.6 Let $0<p \leq 8 / 5, x$ and $y$ be positive real numbers with $x \neq y$. Then

$$
\alpha A^{p}(x, y)+(1-\alpha) L^{p}(x, y)<I^{p}(x, y)<\beta A^{p}(x, y)+(1-\beta) L^{p}(x, y)
$$

holds if and only if $\alpha \leq 1 / 2$ and $\beta \geq(2 / e)^{p}$.

Theorem 5.6 can deduce the following result (see Zhu [15]). 
Corollary 5.7 ([15, Theorem 3]) Let $x$ and $y$ be positive real numbers with $x \neq y$. Then

$$
\alpha A(x, y)+(1-\alpha) L(x, y)<I(x, y)<\beta A(x, y)+(1-\beta) L(x, y)
$$

holds if and only if $\alpha \leq 1 / 2$ and $\beta \geq 2 / e$.

When letting $\alpha=1 / 2$ in the left-hand inequality of (5.11), one can obtain the following result, which is from Sándor $[4,19]$.

$$
I(x, y)>\frac{A(x, y)+L(x, y)}{2} .
$$

Finally, we give the bounds for $L^{p}(x, y)$ in terms of $G^{p}(x, y)$ and $I^{p}(x, y)$, and obtain the following new result.

Theorem 5.8 Let $x$ and $y$ be positive real numbers with $x \neq y$, and $p \geq 286 / 693$. Then

$$
\alpha G^{p}(x, y)+(1-\alpha) I^{p}(x, y)<L^{p}(x, y)<\beta G^{p}(x, y)+(1-\beta) I^{p}(x, y)
$$

holds if and only if $\beta \leq 1 / 2$ and $\alpha \geq 1$.

Theorem 5.8 can deduce a result of Zhu [15]:

Corollary 5.9 ([15, Theorem 4]) Let $x$ and $y$ be positive real numbers with $x \neq y$. Then

$$
\alpha G(x, y)+(1-\alpha) I(x, y)<L(x, y)<\beta G(x, y)+(1-\beta) I(x, y)
$$

holds if and only if $\beta \leq 1 / 2$ and $\alpha \geq 1$.

Obviously, the right-hand side of (5.14) is an extension of the following inequality:

$$
L(x, y)<\frac{1}{2}(G(x, y)+I(x, y))
$$

which was given by Alzer [5].

\section{Competing interests}

The author declares that they have no competing interests.

Received: 24 April 2012 Accepted: 28 November 2012 Published: 18 December 2012

\section{References}

1. Mitrinović, DS: Analytic Inequalities. Springer, Berlin (1970)

2. Ostle, B, Terwilliger, HL: A comparison of two means. Proc. Mont. Acad. Sci. 17, 69-70 (1957)

3. Leach, EB, Sholander, MC: Extended mean values. J. Math. Anal. Appl. 92, 207-223 (1983)

4. Sándor, J: On the identric and logarithmic means. Aequ. Math. 40, 261-270 (1990)

5. Alzer, H: Ungleichungen für Mittelwerte. Arch. Math. 47, 422-426 (1986)

6. Stolarsky, KB: The power mean and generalized logarithmic means. Am. Math. Mon. 87, 545-548 (1980)

7. Zhu, L: Inequalities for hyperbolic functions and their applications. J. Inequal. Appl. 2010, Article ID 130821 (2010)

8. Zhu, L: Some new inequalities for means in two variables. Math. Inequal. Appl. 11(3), 443-448 (2008)

9. Vamanamurthy, K, Vuorinen, M: Inequalities for means. J. Math. Anal. Appl. 183, 155-166 (1994)

10. Anderson, GD, Qiu, S-L, Vamanamurthy, MK, Vuorinen, M: Generalized elliptic integral and modular equations. Pac. J. Math. 192, 1-37 (2000)

11. Pinelis, I: L'Hospital type results for monotonicity, with applications. J. Inequal. Pure Appl. Math. 3, article 5 (2002) (electronic) 
12. Alzer, H, Qiu, S-L: Inequalities for means in two variables. Arch. Math. 80, 201-215 (2003)

13. $\mathrm{Zhu}, \mathrm{L}, \mathrm{Wu}, \mathrm{JH}$ : The weighted arithmetic and geometric means of the arithmetic mean and the geometric mean. J. Math. Technol. 14, 150-154 (1998) (in Chinese)

14. Zhu, L: From chains for mean value inequalities to Mitrinovic's problem II. Int. J. Math. Educ. Sci. Technol. 36, 118-125 (2005)

15. Zhu, L: New inequalities for means in two variables. Math. Inequal. Appl. 11(2), 229-235 (2008)

16. Carlson, BC: The logarithmic mean. Am. Math. Mon. 79, 615-618 (1972)

17. Trif, T: Note on certain inequalities for means in two variables. J. Inequal. Pure Appl. Math. 6, article 43 (2005) (electronic)

18. Sándor, J, Trif, T: Some new inequalities for means of two arguments. Int. J. Math. Math. Sci. 25, 525-532 (2001)

19. Sándor, J: A note on some inequalities for means. Arch. Math. 56, 471-473 (1991)

doi:10.1186/1029-242X-2012-303

Cite this article as: Zhu: New inequalities for hyperbolic functions and their applications. Journal of Inequalities and Applications 2012 2012:303.

Submit your manuscript to a SpringerOpen ${ }^{\circ}$ journal and benefit from:

- Convenient online submission

- Rigorous peer review

- Immediate publication on acceptance

- Open access: articles freely available online

- High visibility within the field

- Retaining the copyright to your article 